\title{
BMJ Open Systematic review and meta-analysis of randomised controlled trials on the effects of potassium supplements on serum potassium and creatinine
}

\author{
Francesco P Cappuccio, ${ }^{1}$ Laura A Buchanan, ${ }^{1}$ Chen $\mathrm{Ji},{ }^{1}$ Alfonso Siani, ${ }^{2}$ \\ Michelle A Miller ${ }^{1}$
}

To cite: Cappuccio FP, Buchanan LA, Ji C, et al. Systematic review and metaanalysis of randomised controlled trials on the effects of potassium supplements on serum potassium and creatinine. BMJ Open 2016;6 e011716. doi:10.1136/ bmjopen-2016-011716

- Prepublication history and additional material is available. To view please visit the journal (http://dx.doi.org/ 10.1136/bmjopen-2016011716).

Received 29 February 2016 Revised 16 June 2016 Accepted 21 June 2016

CrossMark

\begin{abstract}
${ }^{1}$ Division of Health Sciences (Mental Health \& Wellbeing), University of Warwick, WHO Collaborating Centre for Nutrition, Warwick Medical School, Coventry, UK ${ }^{2}$ Institute of Food Sciences, National Research Council, Avellino, Italy
\end{abstract}

Correspondence to Professor Francesco

P Cappuccio;

f.p.cappuccio@warwick.ac.uk

\section{ABSTRACT}

Objectives: High potassium intake could prevent stroke, but supplementation is considered hazardous. We assessed the effect of oral potassium supplementation on serum or plasma potassium levels and renal function.

Setting: We updated a systematic review of the effects of potassium supplementation in randomised clinical trials carried out worldwide, published in 2013, extending it to July 2015. We followed the PRISMA guidelines.

Participants: Any individual taking part in a potassium supplementation randomised clinical trial. Studies included met the following criteria: randomised clinical trials, potassium supplement given and circulating potassium levels reported.

Intervention: Oral potassium supplementation.

Primary outcome measures: Serum or plasma potassium and serum or plasma creatinine.

Results: A total of 20 trials (21 independent groups) were included (1216 participants from 12 different countries). All but 2 were controlled (placebo $n=16$, control $\mathrm{n}=2$ ). . 0 these trials, 15 were crossover, 4 had a parallel group and 1 was sequential. The duration of supplementation varied from 2 to 24 weeks and the amount of potassium given from 22 to $140 \mathrm{mmol} /$ day. In the pooled analysis, potassium supplementation caused a small but significant increase in circulating potassium levels (weighted mean difference (WMD) $0.14 \mathrm{mmol} / \mathrm{L}$, $95 \% \mathrm{Cl} 0.09$ to $0.19, p<1 \times 10^{-5}$ ), not associated with dose or duration of treatment. The average increase in urinary potassium excretion was $45.75 \mathrm{mmol} / 24$ hours, $95 \% \mathrm{Cl} 38.81$ to $53.69, p<1 \times 10^{-5}$. Potassium supplementation did not cause any change in circulating creatinine levels (WMD $0.30 \mu \mathrm{mol} / \mathrm{L}, 95 \% \mathrm{Cl}-1.19$ to $1.78, \mathrm{p}=0.70)$.

Conclusions: In short-term studies of relatively healthy persons, a moderate oral potassium supplement resulted in a small increase in circulating potassium levels and no change in renal function.

\section{BACKGROUND}

A high potassium (K) intake lowers blood pressure (BP) in people with hypertension

\section{Strengths and limitations of this study}

- This is the first study evaluating the safety of increasing potassium intake with supplements on circulating potassium and renal function, based on randomised controlled trials.

- As none of the trials had serum-plasma potassium and creatinine as primary outcomes, the studies may have been underpowered for an effect on these variables.

- The results are only generalisable to the type of patients and individuals who took part in the trials considered (people with hypertension, with and without therapy, normotensives, high-risk groups, non-smokers and random sample of the general population)

- The source of heterogeneity was identified. However, the removal of the heterogeneity did not alter substantially the pooled estimate.

- The study shows that a short-term moderate increase in potassium intake using supplements (average $45 \mathrm{mmol}$ or $1755 \mathrm{mg} /$ day; range 22 $140 \mathrm{mmol}$ or $858-5460 \mathrm{mg} /$ day) is safe and void of risk of hazardous hyperkalaemia or renal deterioration in healthy people and patients, even using blockers of the renin-angiotensin system, whose kidney function is not impaired.

and, to a lesser extent, in people with normal $\mathrm{BP}^{1}{ }^{12}$ However, the beneficial effects of $\mathrm{K}$ extend beyond $\mathrm{BP}$, and it may include a reduction in the risk of stroke (independent of BP changes). ${ }^{134}$

The $\mathrm{K}$ intake in the Western world is relatively low, ${ }^{4}$ and a lower $\mathrm{K}$ intake has been associated with increased risks of cardiovascular disease, especially stroke. ${ }^{1}{ }^{3}$ In randomised controlled trials (RCTs), a moderate increase in $\mathrm{K}$ intake, either as supplement or with diet, reduces $\mathrm{BP},{ }^{12}$ and the $\mathrm{WHO}$ has recently issued global recommendations for a target dietary $\mathrm{K}$ intake of $\geq 90 \mathrm{mmol} /$ day ( $\geq 3510 \mathrm{mg} /$ day) for adults. ${ }^{56}$ 
Different approaches may be adopted to achieve a minimum daily $\mathrm{K}$ intake. ${ }^{7}$ They are dietary modification, use of salt substitutes and $\mathrm{K}$ supplementation. Dietary modifications would involve substituting potassium-low foods with fruits, vegetables, beans and nuts. ${ }^{8}$ They would be appropriate for population-based primary prevention strategies, although it is generally costly and not always equitable. Salt substitutes are commercially available salt mixtures, in which the proportion of sodium chloride is substituted with $\mathrm{K}$ and magnesium salts. ${ }^{9}$ Their use reduces BP and sodium consumption, concurrently increasing $\mathrm{K}$ intake. ${ }^{10}$ This strategy is being considered in China to reduce hypertension. ${ }^{11} 12$ Supplementation with a $\mathrm{K}$ salt represents a cheap intervention to achieve a minimum daily target. ${ }^{1}$ It is acceptable to many patients and has been advocated as a potential cost-effective adjunct in the secondary prevention of stroke. ${ }^{7}$

However, there have been concerns that a widespread moderate increase in $\mathrm{K}$, especially when given as supplementation, could be harmful, leading to hazardous hyperkalaemia. $^{2}{ }^{13-15}$ Furthermore, the proposal of replacing sodium chloride with K-based salt in food manufacturing has also been opposed as potentially harmful. ${ }^{1617}$

Given the potential usefulness and cost-effectiveness of $\mathrm{K}$ supplementation, we decided to update the most comprehensive systematic review carried out to date on the effects of $\mathrm{K}$ supplements on a variety of cardiovascular and biochemical outcomes. ${ }^{1}$ However, at the time neither circulating $\mathrm{K}$ nor creatinine levels were included in the outcomes of interest. We therefore carried out an updated systematic review and a meta-analysis of RCTs of $\mathrm{K}$ supplementation using the same search strategy to estimate the effects that moderate supplementation of $\mathrm{K}$ has on serum levels and renal function.

\section{DATA AND METHODS}

\section{Study selection}

We updated a systematic review carried out as part of a WHO review of the effects of $\mathrm{K}$ on cardiovascular risk factors, published in 2013. ${ }^{1}$ We replicated the search strategy outlined in the paper extending it to papers published from 25 August 2011 to 10 July 2015. The search strategy is shown in online supplementary S1. We kept the same criteria as originally defined. In brief, we planned to include only RCTs (individual and cluster randomised). RCTs must have allocated at least one group of participants to increased K intake (intervention) and one group to lower $\mathrm{K}$ intake (control) for at least 4 weeks. RCTs had to use urinary $\mathrm{K}$ excretion from 24-hour urine collections to estimate actual K intake. The RCTs could not have concomitant interventions (ie, non-drug interventions, antihypertensive drugs or other drugs) in the intervention group unless those interventions were also applied to the control group, so the only difference between the groups was the level of $\mathrm{K}$ intake. We excluded studies targeting acutely ill or HIV-positive people, people admitted to hospital or people with impaired urinary $\mathrm{K}$ excretion due to a medical condition or drug treatment. The searches were run on MEDLINE and Embase with no language restriction. After downloading the abstracts and deleting duplicates, abstracts were scanned. We also included 25 articles selected from the original WHO search. ${ }^{1}$ Studies that met the following inclusion criteria were then selected for the present analysis: (1) RCTs, (2) the intervention group should be K supplement and (3) serum or plasma K levels should be reported. A total of 20 studies were included (13 from the papers published from 2011 to 2015 and 7 from the previous review; figure 1 and see online supplementary S2 and S3). The systematic review and the meta-analysis followed the PRISMA guidelines (see online supplementary Checklist).

\section{Data extraction, risk of bias and quality assessment}

Data extraction was performed in duplicate to counteract human errors and individual biases (LAB and FPC). In addition to extracting relevant data on $\mathrm{K}$ supplementation, serum or plasma $\mathrm{K}$ and creatinine levels, urinary sodium and $\mathrm{K}$ excretions, information was gathered from individual studies to compose a study characteristics table (table 1) which incorporated descriptive and methodological details about the design of the trials, treatment tested and outcomes measured. When data or copies were not available, authors were contacted to provide information. Data extraction sheets were checked by the review team, and differences were resolved by discussion. For the meta-analysis, means and SDs of outcome measures were extracted for the $\mathrm{K}$ supplementation and control groups at the end of each intervention period (for crossover studies), and for baseline and posttreatment period (for parallel-group studies). We assessed the risk of bias quantitatively using the rating scale developed by Downs and Black ${ }^{18}$ (table 1 and see online supplementary S4) and qualitatively using the Cochrane Collaboration's tool ${ }^{19}$ (see online supplementary S5 and S6). For RCTs, we assessed the risk of bias associated with the method of sequence generation (possible selection bias), allocation concealment (possible selection bias), blinding (possible performance bias), selective reporting (possible selective reporting bias), loss to follow-up (possible attrition bias) and completeness of reporting outcome data (possible attrition bias). We rated the risk of bias as being low, unclear or high according to established PRISMA criteria. We used funnel plots to assess the presence of small study bias. We generated 'risk of bias graph' and 'risk of bias summary' figures (see online supplementary S5 and S6).

\section{Data synthesis and analysis}

Comparisons were made between the $\mathrm{K}$ supplementation and control groups with reference to the change between post-treatment groups in crossover trials and differences in changes from baseline to post-treatment groups in parallel-group trials. Changes were calculated such that a positive difference represents an increase 


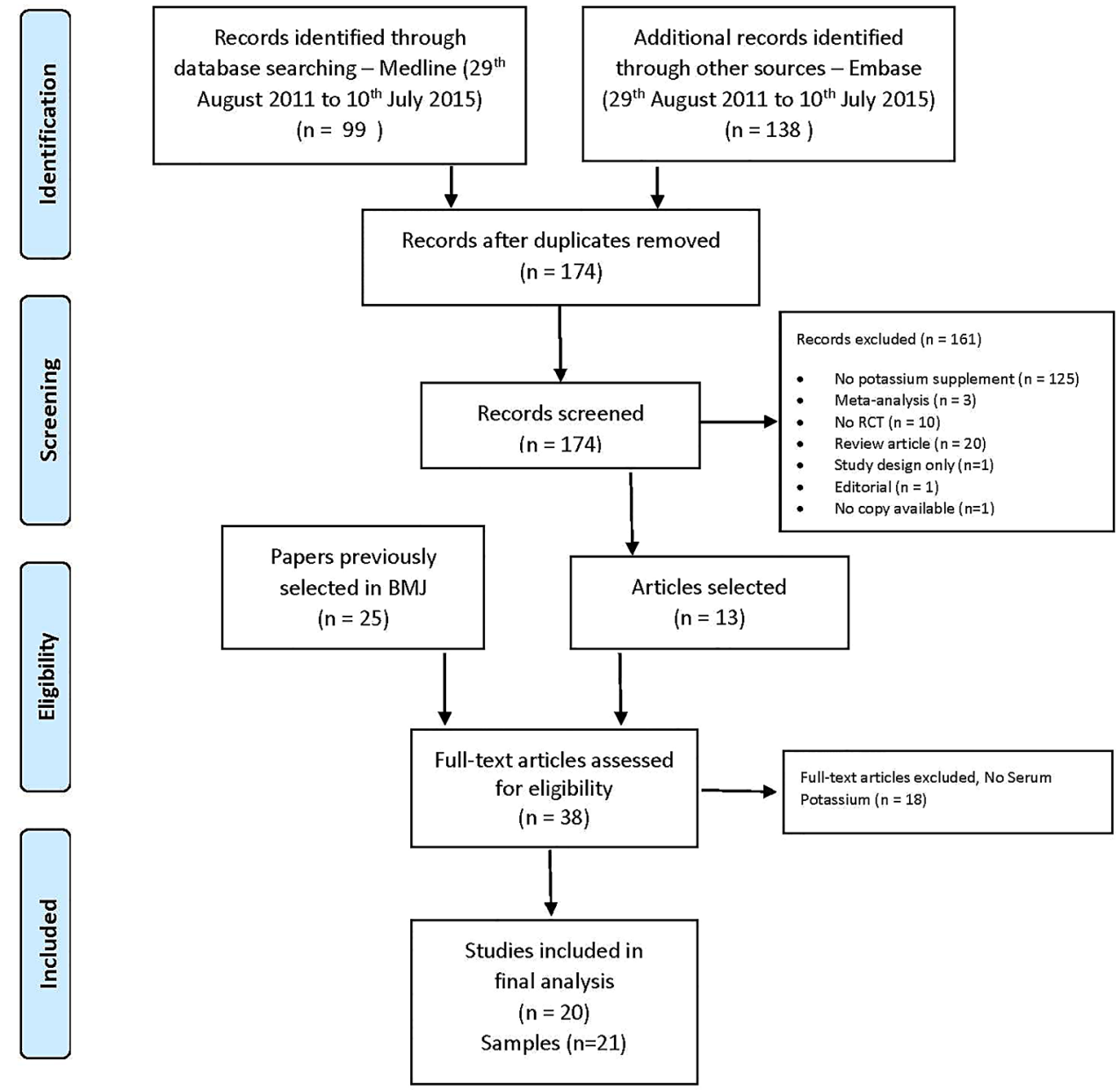

Figure 1 PRISMA flow diagram.

and a negative difference a reduction in the outcome measure in the K-supplemented group compared to placebo or control. Weighted mean differences (WMDs) between the effect of treatment and control were estimated using a random-effect model. ${ }^{20}$ The randomeffects method incorporates an assumption that the different studies are estimating different, yet related, intervention effects. The method is based on the inversevariance approach, making an adjustment to the study weights according to the extent of variation, or heterogeneity, among the varying intervention effects. For each outcome, data from all trials were entered into a funnel plot. Asymmetry of the plot was visually examined and formally tested by Egger's test ${ }^{21}$ to detect publication bias. Statistical heterogeneity among studies was assessed by $\chi^{2}$ test and the $\mathrm{I}^{2}$ statistic. ${ }^{22}$ Comparisons with significant heterogeneity were followed up by sensitivity analysis in which one study was omitted at a time to identify the source of heterogeneity. If dropping the first study did not reduce heterogeneity to a non-significant level, a second study was removed, and so on. Subgroup analyses were also carried out to examine sources of heterogeneity attributable to the study characteristics. Exploratory groups were defined a priori to compare the effect of $\mathrm{K}$ supplementation according to sex, age groups, ethnicity, dose of $\mathrm{K}$ and duration of supplementation. All statistical analyses were performed using RevMan v.5.3.5. and STATA V.14.1.

\section{RESULTS}

Twenty-one samples from the 20 studies were included ${ }^{\text {s1-s20 }}$ (figure 1 and see online supplementary S2). Excluded studies are listed in online supplementary S3.

\section{Characteristics of the trials}

Eighteen studies recruited men and women, whereas two studies recruited only women. ${ }^{\text {s7 s10 }}$ Two studies only studied black participants ${ }^{\mathrm{s} 711}$ (table 1). They included 1216 participants from 12 different countries (6 from UK, 3 from The Netherlands, 2 each from New Zealand and India, 1 from USA, South Africa, Italy, Australia, Kenya, Chile, Japan and Northern Ireland). All but $2^{\mathrm{s} 3}$ s18 were controlled with either placebo $(n=16)$ or a control intervention $(n=2$; table 1$)$. Fifteen used a crossover design, four had a parallel group and one was sequential. All studies used $\mathrm{K}$ chloride as supplement, and one had an additional arm with $\mathrm{K}$ bicarbonate. ${ }^{\text {s17 }}$ The duration of supplementation varied from 2 to 


\begin{tabular}{|c|c|c|c|c|c|c|c|c|c|c|c|c|c|}
\hline $\begin{array}{l}\text { Author } \\
\text { (year) }\end{array}$ & Country & Population & $\begin{array}{l}\text { Participants } \\
\text { (n) }\end{array}$ & $\begin{array}{l}\text { Age } \\
\text { (range), } \\
\text { years }\end{array}$ & Design & Quality* & Control & Potassium & $\begin{array}{l}\text { Duration } \\
\text { (weeks) }\end{array}$ & $\begin{array}{l}\text { Quantity } \\
\text { of K } \\
\text { (mmol/day) }\end{array}$ & $\begin{array}{l}\text { Urinary K } \\
\text { (mmol/day) }\end{array}$ & $\begin{array}{l}\text { Plasma/ } \\
\text { serum K } \\
\text { (mmol/L) }\end{array}$ & Comment \\
\hline $\begin{array}{l}\text { MacGregor } \\
\text { (1982) }\end{array}$ & UK & HPT & $\begin{array}{l}23 \\
12 \text { men; } \\
18 \text { white }\end{array}$ & $45(26-66)$ & RCT-DBX & 25 & Placebo & $\mathrm{KCl}$ (slow-K) & 4 & 64 & $\begin{array}{l}\text { PI: } 62 \\
\text { K: } 118\end{array}$ & $\begin{array}{l}\mathrm{Pl}: 3.84 \\
\mathrm{~K}: 4.02\end{array}$ & \\
\hline $\begin{array}{l}\text { Richards } \\
(1984)\end{array}$ & $\begin{array}{l}\text { New } \\
\text { Zealand }\end{array}$ & HPT & 12 & $(19-52)$ & $\begin{array}{l}\text { RCT-X } \\
\text { (not } \\
\text { blinded) }\end{array}$ & 23 & Control diet & $\mathrm{KCl}$ (elixir) & 4 & 140 & $\begin{array}{l}\mathrm{C}: \sim 60 \\
\mathrm{~K}: \sim 170\end{array}$ & $\begin{array}{l}\text { C: } 3.84 \\
\text { K: } 3.99\end{array}$ & $\begin{array}{l}\text { Control diet } \\
180 \mathrm{Na} / 60 \mathrm{~K}\end{array}$ \\
\hline $\begin{array}{l}\text { Bulpitt } \\
\text { (1985) }\end{array}$ & UK & HPT & $\begin{array}{l}33(K=14 ; \\
C=19) \\
55 \% \text { women }\end{array}$ & 55 & $\begin{array}{l}\text { RCT-P } \\
\text { (open) }\end{array}$ & 21 & $\begin{array}{l}\text { No } \\
\text { supplement }\end{array}$ & $\mathrm{KCl}($ slow-K) & 12 & 64 & $\begin{array}{l}\mathrm{C}: 55 \\
\mathrm{~K}: 95\end{array}$ & $\begin{array}{l}\text { C: } 3.5 \\
\text { K: } 3.8\end{array}$ & $\begin{array}{l}\text { On loop } \\
\text { diuretics }\end{array}$ \\
\hline $\begin{array}{l}\text { Kaplan } \\
(1985)\end{array}$ & USA & $\begin{array}{l}\text { HPT with } \\
\text { hypokalaemia }\end{array}$ & $\begin{array}{l}16 \\
10 \text { women; } \\
13 \text { black }\end{array}$ & $48.8(35-66)$ & RCT-DBX & 25 & Placebo & $\mathrm{KCl}$ & 6 & 60 & $\begin{array}{l}\mathrm{Pl}: 36 \\
\mathrm{~K}: 82\end{array}$ & $\begin{array}{l}\mathrm{Pl}: 3.00 \\
\mathrm{~K}: 3.56\end{array}$ & $\begin{array}{l}\text { Hypokalaemic } \\
\text { on diuretics }\end{array}$ \\
\hline $\begin{array}{l}\text { Smith } \\
(1985)\end{array}$ & UK & HPT & $\begin{array}{l}20 \\
11 \text { men; } 18 \\
\text { white }\end{array}$ & $53(30-66)$ & RCT-DBX & 26 & Placebo & $\mathrm{KCl}$ (slow-K) & 4 & 64 & $\begin{array}{l}\text { PI: } 67 \\
\text { K: } 117\end{array}$ & $\begin{array}{l}\text { Pl: } 3.9 \\
\text { K: } 4.1\end{array}$ & $\begin{array}{l}\text { Reduced } \mathrm{Na} \text { to } \\
70\end{array}$ \\
\hline $\begin{array}{l}\text { Zoccali } \\
\text { (1985) }\end{array}$ & UK & HPT & $\begin{array}{l}19 \\
10 \text { men }\end{array}$ & $\begin{array}{l}\text { M: } 41(26-53) \\
\text { W: } 35(26-53)\end{array}$ & RCT-SBX & 20 & $\begin{array}{l}\text { Placebo } \\
\text { (lactose) }\end{array}$ & KCl (Selora) & 2 & 100 & $\begin{array}{l}\text { PI: } 58 \\
\text { K: } 139\end{array}$ & $\begin{array}{l}\text { Pl: } 3.9 \\
\text { K: } 4.0\end{array}$ & $\begin{array}{l}\text { Selora }(92 \% \\
\mathrm{KCl}, 6 \% \mathrm{~K} \\
\text { gluconate, } 1 \% \\
\text { Ca silicate, } 1 \% \\
\text { glutamic acid) }\end{array}$ \\
\hline $\begin{array}{l}\text { Matlou } \\
\text { (1986) }\end{array}$ & $\begin{array}{l}\text { South } \\
\text { Africa }\end{array}$ & HPT & $\begin{array}{l}32 \\
\text { Black women }\end{array}$ & $51(34-62)$ & RCT-SBX & 21 & $\begin{array}{l}\text { Placebo } \\
\text { (teaspoon } \\
\text { as glucose) }\end{array}$ & $\begin{array}{l}\mathrm{KCl} \text { (teaspoon } \\
\text { as salt) }\end{array}$ & 6 & 65 & $\begin{array}{l}\text { PI: } 52 \\
\text { K: } 114\end{array}$ & $\begin{array}{l}\mathrm{Pl}: 3.87 \\
\mathrm{~K}: 4.32\end{array}$ & \\
\hline $\begin{array}{l}\text { Grobbee } \\
\text { (1987) }\end{array}$ & $\begin{array}{l}\text { The } \\
\text { Netherlands }\end{array}$ & HPT & $\begin{array}{l}40 \\
34 \text { men }\end{array}$ & $(18-28)$ & RCT-DBX & 23 & Placebo & $\mathrm{KCl}($ slow-K) & 6 & 72 & $\begin{array}{l}\text { PI: } 74 \\
\text { K: } 131\end{array}$ & $\begin{array}{l}\mathrm{PI}: 3.76 \\
\mathrm{~K}: 4.00\end{array}$ & Na restriction \\
\hline $\begin{array}{l}\text { Siani } \\
(1987)\end{array}$ & Italy & HPT & $\begin{array}{l}37 \\
23 \text { men }\end{array}$ & $45(21-61)$ & RCT-DBP & 25 & Placebo & $\begin{array}{l}\mathrm{KCl} \\
\text { (Lento-Kalium) }\end{array}$ & 15 & 48 & $\begin{array}{l}\text { Pl: } 57 \\
\text { K: } 87\end{array}$ & $\begin{array}{l}\mathrm{Pl}: 4.4 \\
\mathrm{~K}: 4.3\end{array}$ & \\
\hline $\begin{array}{l}\text { Barden } \\
\text { (1987) }\end{array}$ & Australia & NT & $\begin{array}{l}44 \\
\text { women }\end{array}$ & $(18-55)$ & RCT-DBX & 20 & Placebo & $\mathrm{KCl}($ slow-K) & 4 & 80 & $\begin{array}{l}\mathrm{Pl}: \sim 55 \\
\mathrm{~K}: \sim 115\end{array}$ & $\begin{array}{l}\text { Pl: } 3.725 \\
\text { K: } 3.86\end{array}$ & $\begin{array}{l}\text { Dietary } \mathrm{K} \\
<60 \mathrm{mmol} / \text { day }\end{array}$ \\
\hline $\begin{array}{l}\text { Obel } \\
(1989)\end{array}$ & Kenya & HPT & $\begin{array}{l}48 \\
\text { black }\end{array}$ & 40 & RCT-DBP & 21 & Placebo & $\mathrm{KCl}$ (slow-K) & 16 & 64 & $\begin{array}{l}\text { PI: } 62 \\
\text { K: } 102\end{array}$ & $\begin{array}{l}\mathrm{Pl}: 4.0 \\
\mathrm{~K}: 4.0\end{array}$ & \\
\hline $\begin{array}{l}\text { Patki } \\
\text { (1990) }\end{array}$ & India & HPT & $\begin{array}{l}37 \\
8 \text { men }\end{array}$ & 49.9 & RCT-DBX & 24 & Placebo & $\begin{array}{l}\mathrm{KCl} \text { (Kesol B, } \\
\text { liquid) }\end{array}$ & 8 & 60 & $\begin{array}{l}\text { PI: } 60 \\
\text { K: } 82\end{array}$ & $\begin{array}{l}\text { Pl: } 3.6 \\
\text { K: } 3.7\end{array}$ & \\
\hline $\begin{array}{l}\text { Valdes } \\
\text { (1991) }\end{array}$ & Chile & HPT & 24 & & RCT-DBX & 23 & Placebo & $\mathrm{KCl}$ & 4 & 64 & $\begin{array}{l}\text { PI: } 55 \\
\text { K: } 123\end{array}$ & $\begin{array}{l}\text { Pl: } 3.8 \\
\mathrm{~K}: 4.1\end{array}$ & \\
\hline $\begin{array}{l}\text { Fotherby } \\
\text { (1992) }\end{array}$ & UK & HPT & $\begin{array}{l}18 \\
5 \text { men } \\
\end{array}$ & 75 (66-79) & RCT-DBX & 26 & Placebo & $\mathrm{KCl}$ & 4 & 60 & $\begin{array}{l}\text { Pl: } 60 \\
\text { K: } 99\end{array}$ & $\begin{array}{l}\text { Pl: } 4.3 \\
\text { K: } 4.4\end{array}$ & \\
\hline
\end{tabular}




\begin{tabular}{|c|c|c|c|c|c|c|c|c|c|c|c|c|c|}
\hline $\begin{array}{l}\text { Author } \\
\text { (year) }\end{array}$ & Country & Population & $\begin{array}{l}\text { Participants } \\
\text { (n) }\end{array}$ & $\begin{array}{l}\text { Age } \\
\text { (range), } \\
\text { years }\end{array}$ & Design & Quality* & Control & Potassium & $\begin{array}{l}\text { Duration } \\
\text { (weeks) }\end{array}$ & $\begin{array}{l}\text { Quantity } \\
\text { of K } \\
\text { (mmol/day) }\end{array}$ & $\begin{array}{l}\text { Urinary K } \\
\text { (mmol/day) }\end{array}$ & $\begin{array}{l}\text { Plasma/ } \\
\text { serum K } \\
\text { (mmol/L) }\end{array}$ & Comment \\
\hline $\begin{array}{l}\text { Geleijnse } \\
(1994)\end{array}$ & $\begin{array}{l}\text { The } \\
\text { Netherlands }\end{array}$ & $\begin{array}{l}\text { General } \\
\text { population }\end{array}$ & 100 & $(55-75)$ & RCT-DBP & 24 & $\begin{array}{l}\text { Control } \\
\text { (common } \\
\text { salt) }\end{array}$ & $\begin{array}{l}\mathrm{KCl} \\
\text { (mineral salt) }\end{array}$ & 24 & 22 & $\begin{array}{l}\mathrm{Pl}: 86 \\
\mathrm{~K}: 97\end{array}$ & $\begin{array}{l}\mathrm{Pl}: 4.23 \\
\mathrm{~K}: 4.35\end{array}$ & $\begin{array}{l}\text { Mineral salt: } \\
41 \% \mathrm{KCl}, 17 \% \\
\text { Mg salt, } 1 \% \\
\text { trace minerals }\end{array}$ \\
\hline $\begin{array}{l}\text { Kawano } \\
\text { (1998) }\end{array}$ & Japan & HPT & $\begin{array}{l}55 \\
26 \text { men }\end{array}$ & $62.3(36-77)$ & RCT-DBX & 20 & Placebo & $\mathrm{KCl}$ (slow-K) & 4 & 64 & $\begin{array}{l}\mathrm{Pl}: 54 \\
\mathrm{~K}: 96\end{array}$ & $\begin{array}{l}\mathrm{Pl}: 4.15 \\
\mathrm{~K}: 4.42\end{array}$ & \\
\hline $\mathrm{He}(2010)$ & UK & HPT & $\begin{array}{l}42 \\
30 \text { men }\end{array}$ & $51(18-75)$ & RCT-DBX & 26 & Placebo & $\begin{array}{l}\mathrm{KCl}(\text { slow-K) } \\
\mathrm{KHCO}_{3}\end{array}$ & $4^{4}$ & $\begin{array}{c}64 \\
64\end{array}$ & $\begin{array}{l}\mathrm{Pl}: 77 \\
\mathrm{KCl}: 122 \\
\mathrm{KHCO}_{3}: \\
125\end{array}$ & $\begin{array}{l}\mathrm{Pl}: 4.4 \\
\mathrm{KCl}: 4.6 \\
\mathrm{KHCO}_{3}: 4.4\end{array}$ & \\
\hline $\begin{array}{l}\text { Yusuf } \\
\text { (2012) }\end{array}$ & India & High risk & $\begin{array}{l}518 \\
308 \text { men }\end{array}$ & 57.5 & $\begin{array}{l}\text { RT-Open } \\
\text { label }\end{array}$ & 16 & None & $\mathrm{KCl}$ & 8 & 30 & $\begin{array}{l}- \\
-\end{array}$ & $\begin{array}{l}\text { Pre: } 4.3 \\
\text { K: } 4.4\end{array}$ & \\
\hline $\begin{array}{l}\text { Graham } \\
(2014)\end{array}$ & $\begin{array}{l}\text { North } \\
\text { Ireland }\end{array}$ & $\begin{array}{l}\text { HPT } \\
\text { CVD>10\% }\end{array}$ & $\begin{array}{l}40 \\
32 \text { men }\end{array}$ & $54.8(40-70)$ & RCT-DBX & 23 & Placebo & $\mathrm{KCl}$ (slow-K) & 6 & 64 & $\begin{array}{l}- \\
-\end{array}$ & $\begin{array}{l}\mathrm{PI}: 3.9 \\
\mathrm{~K}: 4.1\end{array}$ & $\begin{array}{l}\text { On doxazosin; } \\
6 \text { weeks } \\
\text { washout }\end{array}$ \\
\hline $\begin{array}{l}\text { Gijsbers } \\
(2015)\end{array}$ & $\begin{array}{l}\text { The } \\
\text { Netherlands }\end{array}$ & Non-smokers & $\begin{array}{l}36 \\
24 \text { men } \\
\text { white }\end{array}$ & $\begin{array}{l}65.8 \\
(47-80)\end{array}$ & RCT-DBX & 25 & Placebo & $\mathrm{KCl}$ capsules & 4 & 72 & $\begin{array}{l}\text { PI: } 55.3 \\
\text { K: } 118.1\end{array}$ & $\begin{array}{l}\text { Pl: } 4.29 \\
\text { K: } 4.41\end{array}$ & Untreated \\
\hline
\end{tabular}

*Downs and Black score (max 27).

C, control; DB, double blind; HPT, hypertension; K, potassium; P, parallel group; PI, placebo; RCT, randomised controlled trial; SB, single blind; X, crossover. 
24 weeks and the amount of $\mathrm{K}$ given from 22 to $140 \mathrm{mmol} /$ day.

\section{The effect of potassium supplementation on plasma} and serum potassium

In the pooled analysis, $\mathrm{K}$ supplementation caused a small increase in plasma or serum $\mathrm{K}$ levels (WMD $0.14 \mathrm{mmol} / \mathrm{L}, 95 \%$ CI 0.09 to $0.19, \mathrm{p}<1 \times 10^{-5}$ ) with no evidence of publication bias (Egger's test $\mathrm{p}=0.65$ ), no asymmetry detected by the 'trim and fill' method (see online supplementary S7) and some heterogeneity between studies $\left(\mathrm{I}^{2}=57 \%, \mathrm{p}=6 \times 10^{-4}\right.$; figure 2$)$. The effects on plasma or serum $\mathrm{K}$ showed a significant variation by dose given (test of subgroup differences: $\mathrm{p}=0.02$ ) (see online supplementary S8). However, it did not appear that there was a dose-dependent effect (meta-regression $\mathrm{p}=0.62$; see online supplementary S9), although most of the doses were in a narrow range of $50-75 \mathrm{mmol} /$ day (see online supplementary $\mathrm{S} 10$ and $\mathrm{S} 11)$. The effect increased from $0.11 \mathrm{mmol} / \mathrm{L} \quad(0.06$, 016) in those without concomitant drug therapy to 0.17 $(0.01,0.34)$ and $0.42(0.17,0.68)$ in those with concomitant drug therapy whether including or excluding ACE-inhibitors (ACE-i) or angiotensin receptor blockers (ARB) (test of subgroup differences: $\mathrm{p}=0.05$; see online supplementary S12). The greatest increase was seen in two studies of hypokalaemic patients in whom the objective was to restore their plasma levels. ${ }^{\mathrm{s} 3}$ s6 The effects were not associated with the geographic area of the world where trials were performed (test of subgroup differences: $\mathrm{p}=0.13$; see online supplementary S13) or the average age of the participants (meta-regression $\mathrm{p}=0.82$; see online supplementary S14). To rule out the influence of poor-quality trials on the overall estimate, we repeated the analysis only in double-blind randomised placebo-controlled crossover trials $(n=12)$. The estimate of effect was $0.19 \mathrm{mmol} / \mathrm{L}$ (95\% CI 0.12 to $\left.0.25, \mathrm{p}<1 \times 10^{-5} ; \mathrm{I}^{2}=42 \%, \mathrm{p}=0.05\right)$. We also estimated the pooled effect in trials of hypertensive patients only ( $\mathrm{n}=16$; WMD $0.19 \mathrm{mmol} / \mathrm{L}, \quad 95 \%$ CI 0.13 to 0.26 , $\left.\mathrm{p}<1 \times 10^{-5} ; \mathrm{I}^{2}=49 \%, \mathrm{p}=0.01\right)$.

\section{The effect of potassium supplementation on urinary potassium excretion}

$\mathrm{K}$ supplementation caused a significant increase in urinary K excretion (WMD $45.75 \mathrm{mmol}(1784 \mathrm{mg}$ ) per day, $95 \%$ CI 37.81 to $53.69, \mathrm{p}<1 \times 10^{-5}$; figure 3 ). The dose of $\mathrm{K}$ had a significant effect on the change in urinary $\mathrm{K}$ (meta-regression $\mathrm{p}=0.001$; figure 4 ). While confirming that the urinary excretion of $\mathrm{K}$ is a good biomarker of $\mathrm{K}$ intake, most studies used between 48 and $100 \mathrm{mmol}$ of $\mathrm{K}$ per day, with only 2 studies of those providing urinary potassium below and above these levels. $^{\text {s2 }}$ s15 When these studies were removed in a sensitivity analysis, the effect remained virtually unchanged (WMD $46.04 \mathrm{mmol}$ (1795 mg) per day, 95\% CI 39.33 to $\left.52.75, \mathrm{p}<1 \times 10^{-5}\right)$.

\section{The effect of potassium supplementation on plasma and serum creatinine}

$\mathrm{K}$ supplementation did not cause any change in renal function as measured by serum or plasma creatinine levels (WMD $0.30 \mu \mathrm{mol} / \mathrm{L}, 95 \%$ CI -1.19 to 1.78 , $\mathrm{p}=0.70$; figure 5). There was some publication bias (Egger's test $\mathrm{p}=0.047$ ); however, the 'trim and fill' method did not show asymmetry in the funnel plot (see

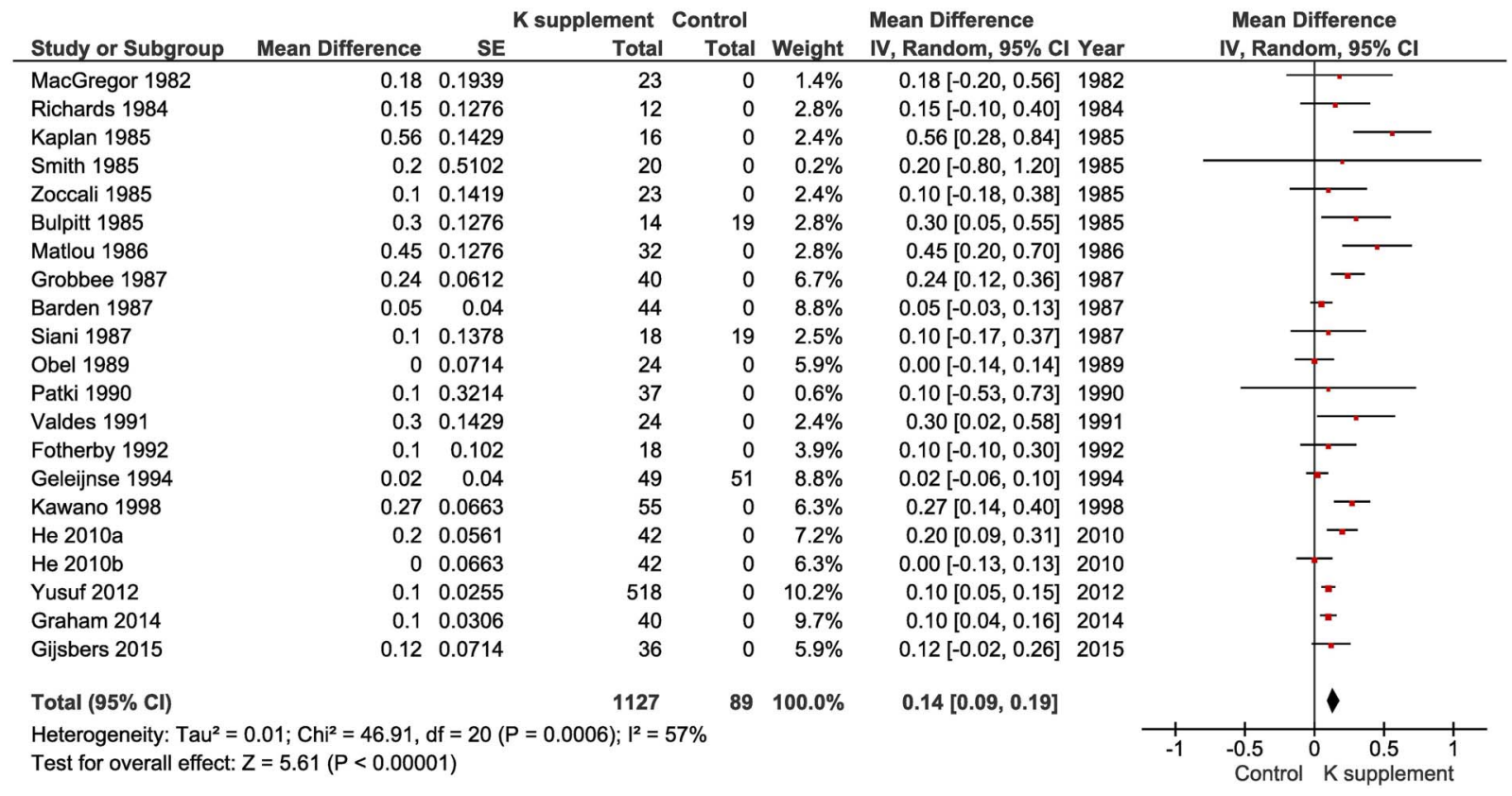

Figure 2 Forest plot of the effect of potassium supplementation on serum or plasma potassium levels in randomised clinical trials. 


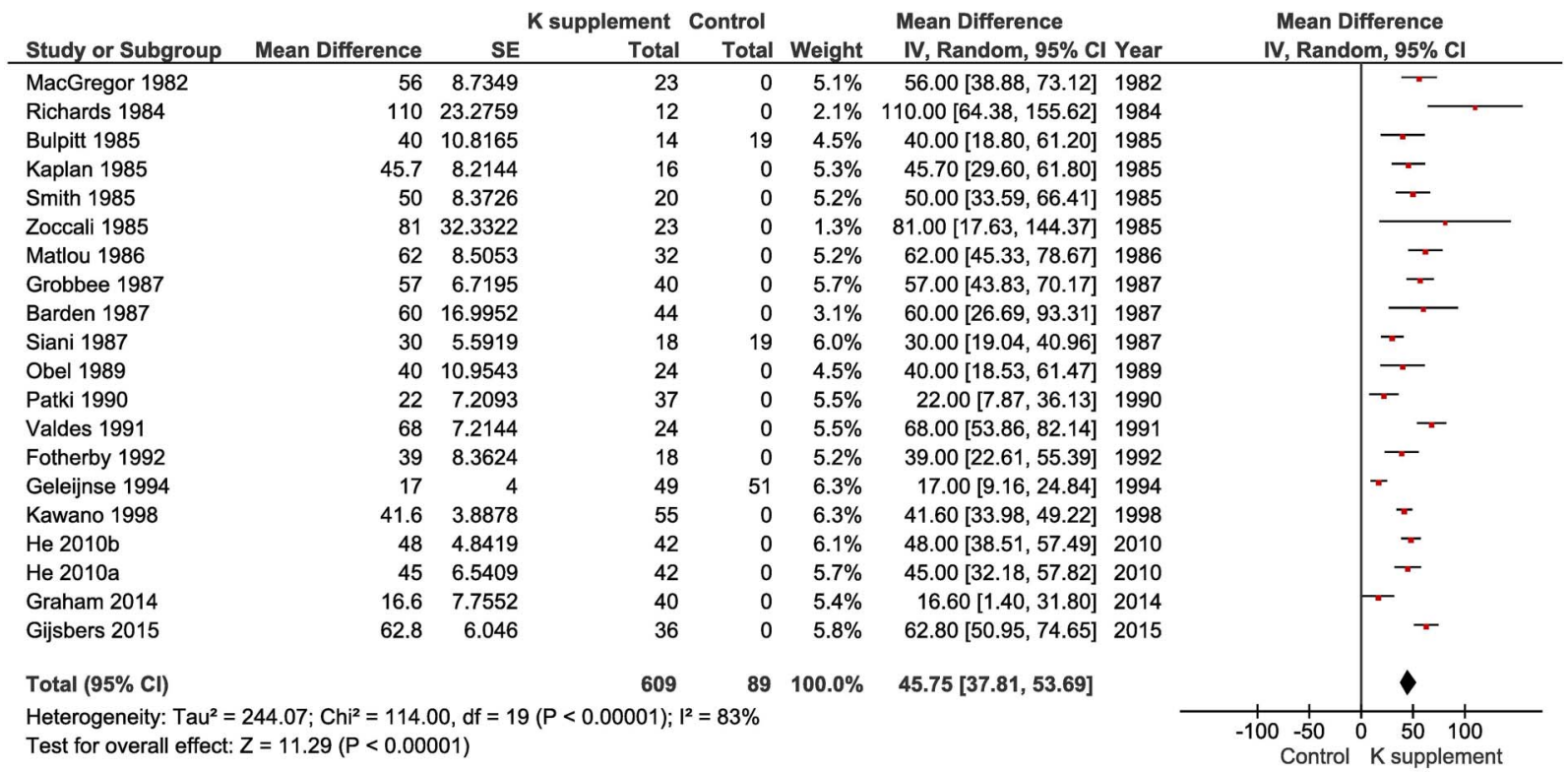

Figure 3 Forest plot of the effect of potassium supplementation on urinary potassium excretion in randomised clinical trials.

online supplementary 15). No heterogeneity was detected $\left(\mathrm{I}^{2}=9 \%, \mathrm{p}=0.35\right)$.

\section{The effect of potassium supplementation on urinary} sodium excretion

$\mathrm{K}$ supplementation did not cause a significant change in urinary sodium excretion (WMD $4.42 \mathrm{mmol}(75.2 \mathrm{mg}$ ) per day, $95 \%$ CI -4.84 to 13.69 , $\mathrm{p}=0.35$ ) (see online supplementary S16). However, there was heterogeneity between studies $\left(I^{2}=55 \%, p=0.003\right)$, fully accounted for by one study (using a salt substitute) ${ }^{\mathrm{s} 15}$. When removed, a small natriuretic effect of $\mathrm{K}$ was detected (WMD $7.42 \mathrm{mmol}$ (126.1 mg) per day, 95\% CI 1.26 to 13.58 , $\mathrm{p}=0.02$; see online supplementary $\mathrm{S} 17) \quad\left(\mathrm{I}^{2}=0 \%, \mathrm{p}=0.86\right)$.

\section{Tolerability}

No serious hazardous side effects were reported in any trial (table 2).

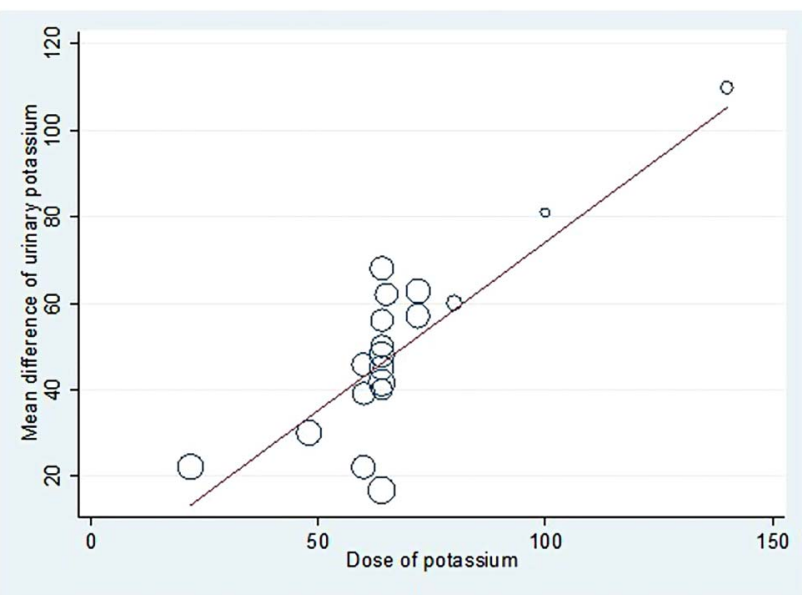

Figure 4 Meta-regression analysis of the changes in urinary potassium excretion for the dose of potassium given.

\section{DISCUSSION}

In the present meta-analysis of RCTs, increasing K intake by moderate $\mathrm{K}$ supplementation (average $45 \mathrm{mmol}$ (1755 mg) per day) causes a small increase in serum $\mathrm{K}$ levels $(0.17 \mathrm{mmol} / \mathrm{L})$ and no change in renal function. The effect on serum $\mathrm{K}$ does not vary with the dose given (from 22 to $140 \mathrm{mmol}$ (from 858 to $5460 \mathrm{mg}$ ) per day), with the duration of the supplementation (2-24 weeks), the presence or absence of concomitant drug therapy, including the presence of blockers of the reninangiotensin system, and with age or geographic location.

To the best of our knowledge, this is the first study evaluating the safety of increasing $\mathrm{K}$ intake with supplements on serum $\mathrm{K}$ and renal function, based on RCTs.

\section{Strengths and limitations}

Our analysis followed the CONSORT guidelines and assessed the risk of bias qualitatively and quantitatively. As none of the trials had been designed to assess the effect of $\mathrm{K}$ supplements on serum $\mathrm{K}$ and creatinine as primary outcomes, the studies may have been underpowered for an effect of these variables. However, given the narrow variability of these measurements and the standard laboratory methods used to determine them, the meta-analytical approach will have compensated by giving more statistical power for the pooled estimates. The source of heterogeneity was identified in three trials. However, their removal, while removing the heterogeneity, did not alter substantially the pooled estimate. These results add to previous evidence of safety of these supplements on other biomarkers like total cholesterol, triglyceride and catecholamine levels. ${ }^{1}$

RCTs and systematic reviews are reliable methods of determining the effects of treatment. ${ }^{23}$ Their usefulness in providing evidence that would influence practice would depend on their internal validity (their quality in 


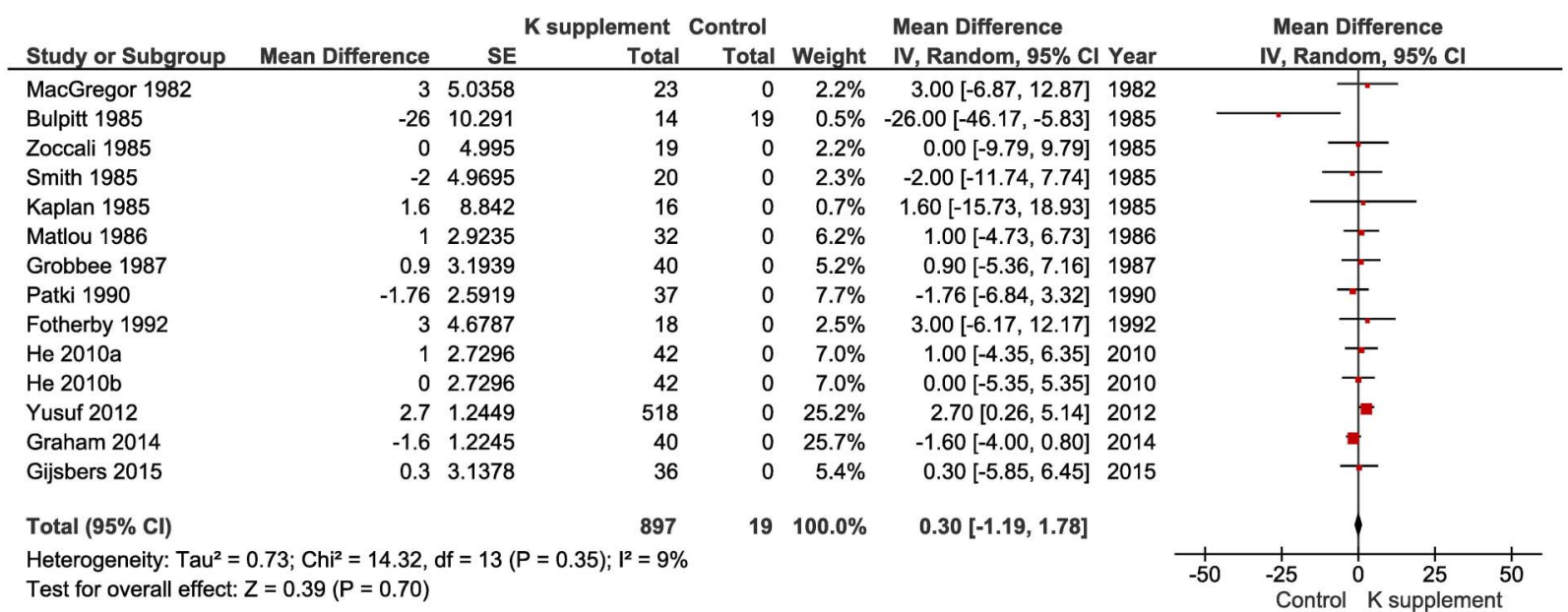

Figure 5 Forest plot of the effect of potassium supplementation on serum or plasma creatinine levels in randomised clinical trials.

design and conduct to minimise erroneous conclusions) and their external validity (their applicability and generalisability in clinical settings). ${ }^{23}{ }^{24}$ Lack of external validity has always been the most frequent criticism by clinicians of RCTs, systematic reviews and guidelines. ${ }^{23}$ It has been argued that the results of trials should be assumed to be externally valid unless there are specific reasons to put this assumption into significant doubt. ${ }^{23}$ Most trials included in the meta-analysis (16 out of 20) were carried out in people with hypertension, while in 2 studies on diuretics and with hypokalaemia. ${ }^{\text {s3 }}$ s6 The remainder were carried out in normotensives, ${ }^{\mathrm{s} 10} \mathrm{a}$ highrisk group, ${ }^{\text {s18 }}$ non-smokers ${ }^{\text {s20 }}$ and in a random sample of the general population. ${ }^{\text {s15 }}$ The results are therefore generalisable to this type of participants only. The reviewed trials had not included people or patients with renal impairment. Sensitivity analyses did not suggest differences according to patients' characteristics like gender, age and type of underlying condition. However, these subgroup analyses have limited margin of interpretation. For instance, only 3 studies were conducted in patients with a mean age of $>60$ years. $^{\text {s14 }}$ s16 ${ }^{\text {s20 }}$ In individual trials, irrespective of dose of $\mathrm{K}$, duration of supplementation or the presence and type of concomitant pharmacological therapy, changes in serum $\mathrm{K}$ were below $0.3 \mathrm{mmol} / \mathrm{L}$, with the exception of 2 studies. ${ }^{\mathrm{s} 6-\mathrm{s} 7}$ In one study, ${ }^{\mathrm{s} 6}$ patients were selected on the basis that they had clinically significant diuretic-induced hypokalaemia; the supplementation increased serum $\mathrm{K}$ by $0.56 \mathrm{mmol} / \mathrm{L}$, restoring it within the normal range. In another study, ${ }^{\mathrm{s}}$ only black women were recruited who had lower $\mathrm{K}$ intake (urinary excretion $52 \mathrm{mmol}$ (2028 $\mathrm{mg}$ ) per day) and a tendency to lower serum $\mathrm{K}(3.87 \mathrm{mmol} / \mathrm{L})$. The supplementation increased serum $\mathrm{K}$ by $0.45 \mathrm{mmol} / \mathrm{L}$ with levels within the normal range.

\section{What is the evidence of hazard?}

Some international bodies discourage the use of $\mathrm{K}$ supplements or K-rich salt replacers as likely cause of hazardous hyperkalaemia. ${ }^{212-17}$ Generally, these warnings, while applied to the general population, are based on case reports referring to either sick patients with end-stage kidney disease ignoring medical advice on $\mathrm{K}$ intake restrictions or excessive intakes leading to increases in serum $\mathrm{K}$ levels of several millimoles per litre with severe hyperkalaemia. ${ }^{25-31}$ In 5 cases, ${ }^{30} 31$ individuals suffered serious hyperkalaemia and in 2 cases fatal hyperkalaemia (serum K 8.9 and $10.8 \mathrm{mmol} / \mathrm{L}$ ) following $\mathrm{K}$ overdoses of up to $723 \mathrm{mmol}(28200 \mathrm{mg})$ in a day. In other reports, ${ }^{25} 262829$ individuals with severe renal impairment and, in some cases, already of haemodialysis ${ }^{28}$ abused K-containing salt substitutes. Finally, 2 Afro-Caribbean men on antihypertensive therapy including ACEinhibitors $^{27}$ presented with serum $\mathrm{K}$ levels of 7.6 and $7.0 \mathrm{mmol} / \mathrm{L}$ following daily use of $70 \mathrm{mmol}(2730 \mathrm{mg})$ and $133 \mathrm{mmol}(5187 \mathrm{mg}$ ) of $\mathrm{K}$ added to their food daily as a salt substitute. We agree that caution should be exercised in people with severe impairments of their renal function when considering potassium supplements. However, our analysis suggests that a more 'moderate' supplement does not seem to cause severe hyperkalaemia or deterioration in renal function in people with normal renal function, even in the presence of drugs that block the renin-angiotensin-aldosterone system.

\section{Benefits of potassium supplements}

The consideration of the use of moderate increases in $\mathrm{K}$ intake with supplements is encouraged by the evidence of potential benefits in controlling hypertension, ${ }^{1}{ }^{4-6}$ a surrogate end point for cardiovascular risk, stroke in particular. While the evidence of a potential benefit of $\mathrm{K}$ on stroke prevention in humans is mainly derived from observational cohort studies, ${ }^{1}{ }^{3-6}$ early animal experiments indicated that the reduction in mortality of strokeprone hypertensive rats given $\mathrm{K}$ supplements was seen even in BP-matched animals, ${ }^{32} 33$ suggesting that, in rats, $\mathrm{K}$ reduces stroke rates also through mechanisms other than BP reduction. Subsequent evidence showed a protective vascular effects of $\mathrm{K}$, especially in thrombus formation. ${ }^{7}$ High $\mathrm{K}$ increases the lumen of cerebral 
Table 2 Tolerability of potassium supplementation and reported adverse reactions in the published papers

\begin{tabular}{|c|c|}
\hline Study & Description \\
\hline MacGregor (1982) & All patients who entered the trial completed it without any adverse effect. \\
\hline Richards (1984) & The study was completed without incident. \\
\hline Bulpitt (1985) & $\begin{array}{l}\text { The patients were asked about any symptom of indigestion. Three reported of the symptom before } \\
\text { being given } \mathrm{K} \text { supplementation but not afterwards, and one patient in the control group lost this } \\
\text { symptom. One patient in each group started to complain of a decrease in appetite at the end of the trial. }\end{array}$ \\
\hline Kaplan (1985) & None of the 16 patients had notable changes in clinical status or symptoms during the 16 -week trial. \\
\hline Smith (1985) & Nothing reported. No withdrawals. \\
\hline Zoccali (1985) & $\begin{array}{l}\text { Four patients were withdrawn, one because the first treatment (Selora salt) precipitated diarrhoea, two } \\
\text { because they were unable to tolerate the taste of the } \mathrm{K} \text { preparation and one because the diastolic } \mathrm{BP} \\
\text { after the placebo phase had risen to } 120 \mathrm{~mm} \mathrm{Hg} \text {. Twelve patients interviewed after the study } \\
\text { commented on the unpleasant taste of the } \mathrm{K} \text { preparation. }\end{array}$ \\
\hline Matlou (1986) & $\begin{array}{l}\text { Three patients dropped out, one was admitted to hospital with an intercurrent illness and was given } \\
\text { moduretic, and two failed consistently to keep their appointments. }\end{array}$ \\
\hline Grobbee (1987) & Nothing reported. All participants entering the double-blind study completed it. \\
\hline Siani (1987) & All patients completed the trial without suffering any adverse effects. \\
\hline Barden (1987) & Forty-three women completed the trial, with one withdrawing during the first treatment period. \\
\hline Obel (1989) & $\begin{array}{l}\text { All } 48 \text { patients completed the trial. None developed any notable untoward effect attributable to } \\
\text { medication. }\end{array}$ \\
\hline Patki (1990) & $\begin{array}{l}\text { Three patients given placebo, four given } \mathrm{K} \text {, and four given } \mathrm{K} \text { and } \mathrm{Mg} \text { reported of pain in the abdomen } \\
\text { and nausea, but this passed off and did not require withdrawal of treatment. }\end{array}$ \\
\hline Valdes (1991) & Nothing reported. \\
\hline Fotherby (1992) & $\begin{array}{l}\text { There were no withdrawals from the study. All patients took at least } 90 \% \text { of the trial medication }(\ldots) \\
\text { which was well tolerated with no reported adverse effects. }\end{array}$ \\
\hline Geleijnse (1994) & $\begin{array}{l}\text { Complete follow-up was achieved by } 97 \text { of the } 100 \text { randomised participants. Two of the controls } \\
\text { withdrew after } 8 \text { and } 16 \text { weeks because of admission to hospital for symptoms not related to } \\
\text { intervention. One person withdrew in the mineral salt group after } 6 \text { weeks because of dislike of the } \\
\text { foods. (...) Reports of side effects and lifestyle changes during intervention were minimal and equally } \\
\text { distributed among the study groups. }\end{array}$ \\
\hline Kawano (1998) & One patient withdrew due to gastrointestinal symptoms during $\mathrm{K}$ supplementation. \\
\hline $\mathrm{He}(2010)$ & Four patients withdrew from the study (reasons not given). \\
\hline Yusuf (2012) & $\begin{array}{l}\text { Twenty-seven (10.5\%) of those receiving } \mathrm{K}+\text { supplements permanently discontinued this ( } 11 \text { for } \\
\text { dyspepsia, } 4 \text { for elevated creatinine or } \mathrm{K}+, 12 \text { for other reasons). }\end{array}$ \\
\hline Graham (2014) & $\begin{array}{l}\text { The main side effect was that of gastrointestinal irritation. This was reported by four participants, while } \\
\text { taking potassium chloride. Symptoms resolved with a reduction in potassium supplementation from } \\
64 \mathrm{mmol} / 4.8 \mathrm{~g} \text { ( } 8 \text { tablets) to } 48 \mathrm{mmol} / 3.6 \mathrm{~g} \text { daily ( } 6 \text { tablets). No participant withdrew from the study as a } \\
\text { result of this side effect. }\end{array}$ \\
\hline Gijsbers (2015) & $\begin{array}{l}\text { Reported side effects in participants' diaries indicated that } 19 \text { persons experienced gastrointestinal } \\
\text { symptoms during sodium, } 21 \text { during potassium and } 8 \text { during placebo supplementation }(P=0.004) \text {. Other } \\
\text { side effects including dizziness, headache, illness, shortness of breath and oedema were not } \\
\text { significantly different among the three treatments. }\end{array}$ \\
\hline
\end{tabular}

arteries, ${ }^{34}$ inhibition of atherosclerotic vascular lesions, decreases vascular smooth muscle cell proliferation and migration, decreases free radical formation, reduces LDL-cholesterol oxidation and decreases platelet aggregation. ${ }^{35}$ These results have supported recent calls for considering trials of a moderate increase in $\mathrm{K}$ intake with supplements to test whether this is a viable and potentially cost-effective strategy to prevent stroke incidence in high-risk groups and stroke recurrence. ${ }^{7}$

\section{How can we increase potassium intake?}

Increasing $\mathrm{K}$ intake through diet is an efficacious method to lower $\mathrm{BP}^{37}$ It would involve increasing the consumption of K-rich foods like fruits, vegetables, beans and nuts. ${ }^{8}$ While the dietary approach is the basis of public health programmes promoting healthy eating, it is generally costly and difficult to implement in low socioeconomic groups ${ }^{38} 39$ as well as among vulnerable patients at high risk, like elderly people who may find it difficult to change their diet, and would widen health inequalities. The addition of $\mathrm{K}$ through salt substitutes or supplements is an alternative cheap intervention to achieve daily targets. The quantity of $\mathrm{K}$ can be titrated more precisely, and it is acceptable to many people. Caution should be exercised in people who suffer from kidney disease and have renal dysfunction, or in those who may be on antialdosterone therapy.

\section{CONCLUSIONS}

The present study shows that a moderate increase in $\mathrm{K}$ intake using supplements could be safe and void of risk of hazardous hyperkalaemia or renal deterioration in 
people and patients whose kidney function is not impaired, even using blockers of the renin-angiotensin system. Given the limitations of the analysis to younger patient groups and short-term supplementations, and the potential benefit as an adjunct preventive strategy, ${ }^{7}$ these results should encourage feasibility trials to ascertain the generalisability of these findings to patients with normal renal function at high risk of stroke.

Acknowledgements The work has been carried out under the remit of the WHO Collaborating Centre for Nutrition (UNK-257). However, the publication does not necessarily represent the decisions or the stated policy of WHO and the designations employed and the presentation of material do not imply the expression of any opinion on the part of WHO.

Contributors FPC developed the idea, supervised the analysis and drafted the manuscript. $L A B$ and $C J$ jointly developed the analytical approach to data handling and carried out the analysis. AS and MAM contributed to discussions in the selection, analysis and discussion of the results. All authors contributed to the final version of the manuscript. FPC is the guarantor.

Funding LAB was supported by an Undergraduate Research Support Scheme bursary from the University of Warwick.

Competing interests FPC is an unpaid member of CASH, WASH, the UK National Forum and the UK Public Health NACD; unpaid technical advisor to NICE, the WHO Geneva and Office for Europe, EMRO Region and the Pan American Health Organization; and unpaid Vice-President and Trustee of the British Hypertension Society and Trustee of the Student Heart Health Charity.

Provenance and peer review Not commissioned; externally peer reviewed.

Data sharing statement No additional data are available.

Open Access This is an Open Access article distributed in accordance with the Creative Commons Attribution Non Commercial (CC BY-NC 4.0) license, which permits others to distribute, remix, adapt, build upon this work noncommercially, and license their derivative works on different terms, provided the original work is properly cited and the use is non-commercial. See: http:// creativecommons.org/licenses/by-nc/4.0/

\section{REFERENCES}

1. Aburto NJ, Hanson S, Gutierrez H, et al. Effect of increased potassium intake on cardiovascular risk factors: systematic review and meta-analyses. BMJ 2013;346:f1378

2. Food Standards Australia New Zealand. Systematic review of the evidence for a relationship between potassium and blood pressure. 2014:1-28.

3. D'Elia L, Barba G, Cappuccio FP, et al. Potassium intake, stroke and cardiovascular disease: a meta-analysis of prospective studies. J Am Coll Cardiol 2011;57:1210-19.

4. He FJ, MacGregor GA. Beneficial effects of potassium. BMJ 2001;323:497-501.

5. World Health Organization. Effects of increased potassium intake on blood pressure, renal function, blood lipids, and other potential adverse effects. Geneva: WHO, 2012:1-122.

6. World Health Organization. Guideline: potassium intake for adults and children. Geneva: WHO, 2012;1-42.

7. Hunt BD, Cappuccio FP. Potassium intake and stroke risk: a review of the evidence and practical considerations for achieving a minimum target. Stroke 2014:45:1519-22.

8. Sacks FM, Svetkey LP, Vollmer WM, et al, DASH-Sodium Collaborative Research Group. Effects on blood pressure of reduced dietary sodium and the Dietary Approaches to Stop Hypertension (DASH) diet. DASH-Sodium Collaborative Research Group. N Engl J Med 2001;344:3-10.

9. Peng YG, Li W, Wen XX, et al. Effects of salt substitutes on blood pressure: a meta-analysis of randomized controlled trials. Am J Clin Nutr 2014;100:1448-54.

10. Binia A, Jaeger J, Hu Y, et al. Daily potassium intake and sodium-to-potassium ratio in the reduction of blood pressure: a meta-analysis of randomized controlled trials. J Hypertens 2015;33:1509-20.
11. The China Salt Substitute Study Collaborative Group. Salt substitution: a low-cost strategy for blood pressure control among rural Chinese. A randomized, controlled trial. J Hypertens 2007;25:2011-18.

12. Zhou B, Wang HL, Wang WL, et al. Long-term effects of salt substitution on blood pressure in a rural North Chinese population. $J$ Hum Hypertens 2013;27:427-33.

13. Expert Group on Vitamins and Minerals. Safe upper levels of vitamins and minerals. Food Standard Agency, 2003.

14. Institute of Medicine Food and Nutrition Board. Dietary reference intake: water, potassium, sodium, chloride, and sulphate. Washington DC, USA: Institute of Medicine, National Academy Press, 2005.

15. EFSA. Opinion of the Scientific Panel on Dietetic Products, Nutrition and Allergies on a request from the Commission related to the tolerable upper intake level of potassium (Request N. EFSA-Q2003-018). EFSA J 2005;193:1-19.

16. Committee on Toxicity. Potassium-based salt replacers: current status of COT consideration. 2014. http://cot.food.gov.uk/sites/ default/files/cot/cotpapotas.pdf (accessed 19 Jan 2016)

17. Committee on Toxicity of Chemicals in Food, Consumer Products and the Environment. Second draft statement on potassium-based replacements for sodium chloride and sodium-based additives (TOX/ 2015/11). http://cot.food.gov.uk/sites/default/files/TOX-2015-11_0. pdf (accessed 19 Jan 2016).

18. Downs SH, Black N. The feasibility of creating a checklist for the assessment of the methodological quality both of randomised and non-randomised studies of healthcare interventions. J Epidemiol Community Health 1998;52:377-84.

19. Higgins JPT, Altman DG, Gotzsche PC, et al. The Cochrane Collaboration's tool for assessing risk of bias in randomised trials. BMJ 2011;343:d5928.

20. DerSimonian R, Laird N. Meta-analysis in clinical trials. Control Clin Trials 1986;7:177-88.

21. Egger M, Davey SG, Schneider M, et al. Bias in meta-analysis detected by a simple, graphical test. BMJ 1997;315:629-34.

22. Higgins JP, Thompson SG, Deeks JJ, et al. Measuring inconsistency in meta analyses. BMJ 2003;327:557-60.

23. Rothwell PM. External validity of results of randomized trials: disentangling a complex concept. Int J Epidemiol 2010;39:94-6.

24. Dekkers OM, von Elm E, Algra A, et al. How to assess the external validity of therapeutic trials: a conceptual approach. Int J Epidemiol 2010;39:89-94.

25. Hoyt RE. Hyperkalemia due to salt substitutes. JAMA 1986;256:1726

26. van der Loeff HJS, van Schijndel RJMS, Thijs LG. Cardiac arrest due to oral potassium intake. Int Care Med 1988;15:58-9.

27. Ray KK, Dorman S, Watson RDS. Severe hyperkalaemia due to the concomitant use of salt substitutes and ACE inhibitors in hypertension: a potentially life threatening interaction. J Hum Hypertens 1999;13:717-20.

28. Doorenbos CJ, Vermeij CG. Danger of salt substitutes that contain potassium in patients with renal failure. BMJ 2003;326:35-7.

29. Dent A, Walmsley D, Dhandapani S. Hyperkalaemia is a risk with low sodium salt in vulnerable patients. BMJ 2011;343:d4514.

30. Wettli CW, Davis JH. Fatal hyperkalemia from accidental overdose of potassium chloride. JAMA 1978;240:1339.

31. Illingworth RN, Proudfoot AT. Rapid poisoning with slow-release potassium. BMJ 1980;281:485-6.

32. Tobian L, Lange J, Ulm K, et al. Potassium reduces cerebral hemorrhage and death rate in hypertensive rats, even when blood pressure is not lowered. Hypertension 1985;7:I-110-14.

33. Tobian L, Lange JM, Ulm KM, et al. Potassium prevents death from strokes in hypertensive rats without lowering blood pressure. J Hypertens 1984;2:S363-6.

34. Rigsby CS, Pollock DM, Dorrance AM. Dietary potassium supplementation improves vascular structure and ameliorates the damage caused by cerebral ischemia in normotensive rats. Nutr Metab (Lond) 2008;5:3.

35. Young DB. Role of potassium in preventive cardiovascular medicine. Norwell, MA: Kluwer Academic Publishers, 2001.

36. Young DB, Ma G. Vascular protective effects of potassium. Semin Nephrol 1999;19:477-86.

37. Siani A, Strazzullo P, Giacco A, et al. Increasing the dietary potassium intake reduces the need for antihypertensive medications. Ann Intern Med 1991;115:753-9.

38. Steptoe A, Perkins-Porras L, McKay C, et al. Behavioural counselling to increase consumption of fruit and vegetables in low income adults: randomised trial. BMJ 2003;326:855-8.

39. Cappuccio FP, Rink E, Perkins-Porras L, et al. Estimation of fruit and vegetable intake using a two-item dietary questionnaire: a potential tool for primary healthcare workers. Nutr Metab Cardiovasc Dis 2003;13:12-19. 